\title{
Wireless Sensor Network to Support home Care in TELEMEDICINE APPLICATIONS
}

\author{
AUTHORS \\ Silvia Jiménez-Fernández ${ }^{1}$, Antonio Cobo-Sánchez-de-Rojas ${ }^{1}$, Álvaro Araujo-Pinto ${ }^{2}$, Pedro \\ Malagón ${ }^{2}$, Octavio Nieto-Taladriz ${ }^{2}$, Paula de Toledo ${ }^{1}$ and Francisco del Pozo ${ }^{1}$ \\ ${ }^{1}$ Grupo de Bioingeniería y Telemedicina. Universidad Politécnica de Madrid. \\ ${ }^{2}$ Laboratorio de Sistemas Integrados. Universidad Politécnica de Madrid.
}

\section{INTRODUCTION}

The care of patients suffering from chronic diseases is a growing source of expense for healthcare services around the world. The implementation of new models for patients' treatment and follow-up needs to be faced in order to increase patients' quality of life, and to reduce the costs associated. In this paper we propose a point-of-care for home care scenarios that is based on the remote monitoring of biomedical parameters.

\section{BACKGROUND}

Research experiences in the past have proved that home care can maintain or even improve old or chronically ill people's quality of life, as well as decrease healthcare costs associated. Home care delivery can still be improved through advances in human-machine interfaces that enable users (i.e. mostly old people) to deal easily and non-intrusively with technology (Ades 2000, Hernandez 2003, Morlion 2002). Many limitations brought on by aging are expected to be overcome by innovation in the area of Ambient Intelligence (Aml) (Cucchiara 2005, Trivedi 2005), and focused on the design of devices easy to use and easy to learn that work transparently and pervasively to aid the user (Remagnino 2005).

Therefore, next generation home care and follow-up systems have important challenges to face, such as the development of non-intrusive systems that provide the right medical services on a highly-usable basis or systems that require minimum human-machine interaction to promote an augmented environment while making computers disappear in the background. Of course, it is important to make these necessities possible by means of developing affordable solutions for public and private healthcare institutions, only achievable with low cost structures easy to install and to maintain.

In order to achieve these requirements, we propose a Wireless Sensor Network, based on a Home Area Network (HAN), featuring a gateway, several biomedical sensors (pulse oximeter, electrocardiograph and accelerometer to quantify daily activity) that the doctor recommends to the patient to control his/ her health and a user interface, key element to inform the user of events registered (such as start of the exam, low battery supply, etc). All network nodes are provided of wireless capabilities to allow full mobility (ISTAG 2002).

\section{The Biomedical Wireless Sensor Network}

\section{REQUIREMENTS}

The requirements taken into account when designing the home care point-of-care have been, mainly: self-management, context awareness, plug-and-play features and security. Due to the need to achieve wearable and low cost sensors limited resources in processing power have also been taken into account.

Regarding self-management and plug-and-play capabilities, the point-of-care has been designed to, automatically, route data packets using neighbor nodes as well as to adapt its topology to network changes. That is to say that the user does not have to consider nodes' breakup, or the addition of new nodes/sensors to the network, as they are transparently recognized and managed. For security reasons, data coming from new sensors is not sent to the telemedicine server until approved by it. 
The nodes of the wireless network have to be context aware to adapt their behavior accordingly to environmental variables that govern the system: such as the actor of the system and his/ her preferences, moment of the day when usage takes place, season, etc.

Once again, it is important to achieve these goals by means of low cost systems, as these points-of-care will not be deployed massively or used in clinical routine if they are not costefficient and provide benefits over traditional healthcare delivery. Of course, as costs are reduced, limitations arise, such as processing power, tight memory sizes, or low power consumption (batteries are required to last long enough to allow several measures to take place, as well as to support the wireless radio link power consumption).

Above all, there are security issues to be considered. For real scenario setups, where sensitive and personal data will be in transit through the network, refusing intrusions, avoiding denial of service, driving the system into a safe state in case of failure, etc. are a must.

\section{FUNCTIONALITY}

Based on the requirements stated above, the wireless sensor network consists of three different elements: a gateway (center of the point-of-care), the sensors (used to monitor the patient's vital signs), and the user interface (to inform of upcoming exams, problems, etc).

The functionality of the different elements follows. The gateway has twofold capability: 1) it is used to communicate with the sensors that the patient has, and 2) it is needed to send data to and from the healthcare institutions (i.e. hospital, primary care, etc.). The information regarding the doctor's prescription of medical exams is sent to the gateway, where it is stored, processed and routed to the specific sensor, stating the duration, sample rate, quality of the measure, amplification factor, or any precise parameter needed to collect the data.

The sensors have been developed as stand alone wireless devices and have been provided of automatic start of the measure, auto-discovery, and auto-configuration facilities. That is to say that the patient does not need to take care of configuring nor connecting them, for instance, to upload or download the data collected. It is important to note that the user does not need to ensure either that the sensors are powered on and off, as they are suspended and resume automatically after a given time. When resume is done, every half minute, several tasks take place:

1) Checking the state of the sensor to become aware of the patient's willingness to start an exam. When the user wants to commence it, he/ she places the sensor on, so that the device is able to collect data. This situation takes place no matter if the doctor programmed the test or if the patient decided to take it by himself/ herself (because not feeling alright).

2) Connecting to the gateway. The radiofrequency module is powered on and a "keep alive" message is sent to the gateway. If there is information that needs to be transmitted to the node, as imminent exams that need to take place, it is sent at this moment. Afterwards, if data are still stored in the sensor, they are forwarded to the gateway.

3) Checking battery level. When batteries are close to be used up, an alarm is sent to the gateway and the user is notified so that he/she can recharge them. This check is performed every ten minutes instead of every half minute.

4) Checking network failures.

Once all these tasks have been performed, the device is suspended again to save batteries. Using this method, battery consumption is decreased up to a maximum of 60 percent.

\section{RESULTS}

The abovementioned approach has led to the definition and development of a home point-ofcare that aims to foster independent living of the elderly or chronically ill featuring high usability and minimum human machine interaction and remote monitoring.

This has been achieved using a gateway to route information from the house to the outside institutions (hospitals, private institutions, etc.), and also within the home environment, through a network of monitoring sensors. In order to minimize maintenance and installation costs, as well as to improve usability, a wireless network (LR-WPAN) has been used to interconnect the sensors and the hub (J imenez-Fernandez 2006). 
Each node (i.e. sensing device) has three different parts. First, the biomedical sensor itself: an off-the-shelf OEM board (ECG, pulse oximeter) or an ad-hoc module (accelerometer) that has been provided of a second component, an autonomous power supply. As the power requirements of each sensor are different, customized supply stages have been designed using a standard interface with commercial AAA batteries, and always looking for the highest efficiency that maximizes sensors' life time. Finally, the third part that has been added provides the "intelligence" explained in the requirements section: awareness of patient's willingness to perform a measurement, network functionality (e.g. routing, reconfiguration), etc. The main component of this stage is a radio transceiver in the ISM band of $2.4 \mathrm{GHz}$ that implements the physical layer and the MAC layer of the IEEE 802.15.4 wireless standard. The main characteristics featured are:

- Possible bit rates: $250 \mathrm{Kbps}, 40 \mathrm{Kbps}$ or $20 \mathrm{Kbps}$. We are using $250 \mathrm{Kbps}$.

- Possible network topologies: mesh, star or point to point. We are using star topology because it is more efficient for this propose.

- Support for low latency devices.

- Channel access using CSMA-CD (Carrier Sense Multiple Access/Collision Detection).

- Low power consumption.

Therefore, our network requirements and functionality suit perfectly with the given standard. In order to implement the remaining layers of the protocol stack a low-cost PIC 16F microcontroller that connects to the radio transceiver through a SPI communication interface has been attached to each node. The commonly used OSI architecture has been refused in favor of an architecture based on services. The goal pursued with this design is the implementation of applications that arise as a mere aggregation of services and relations between services, rather than a node-focused orientation, simplifying the development of user applications (Sgroi 2003). Our services are built on top of a communication middleware (Coleri 2003, Heinzelman 2004, Ranganathan 2003) that abstracts network or hardware specific tasks to the application. This middleware allows sending data to any node of the network without considering its location, sending broadcast messages, etc, and all this happens using a simple and unique interface (based on query-response) that can be dynamically instantiated in any node at any time. This allows to easily compose applications taking into account many complex aspects, such as privacy, safety, load balancing, low energy consumption, etc (Coleri 2003, Rabaey 2000), and in a totally transparent way to the user. The protocol stack can be depicted in figure 1 .

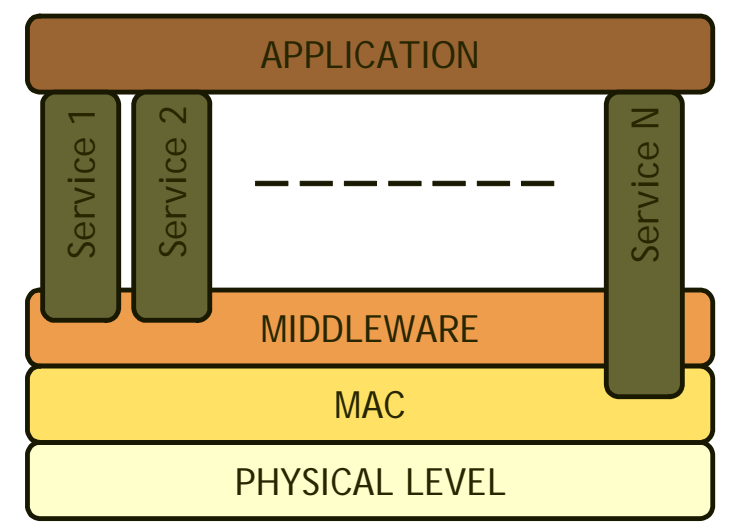

Figure 1. Protocol stack

The idea behind all this approach is to isolate common behavior that can be reused from other applications and to encapsulate it as system services, so that once the system is running the addition of a new application becomes easy.

Another important fact that has to be emphasized is that, in contrast to traditional architectures, service oriented architectures do not force all services to access all middleware/layers available (as can be seen in figure 1). For instance, in order to check battery levels, communication protocols can be bypassed and access to physical level directly used, making signal processing more efficient. 
All in all, an interoperable, reliable system, abstracting the hardware from the applications has been designed, developed and implemented. It is important to highlight that the size of the prototype is not comparable to that of wearable devices, but the aim of this work was just to emulate the behavior that wearable sensors will have in the future, not to miniaturize existing ones.

\section{FUTURE TRENDS}

This paper has presented a system that addresses usability issues, plug-and-play interoperability, reconfigurability and wireless communication between the different devices of the home's point-of-care. Emerging trends on the field of research are focused on applying standards to these issues in order to facilitate vendor interoperability and so, avoid closed solutions. At the moment there is a family of standards, ISO11073 / IEEE 1073, that aims to increase interoperability at the hospital's point-of-care. Although it is not specifically designed to undertake the problem of integrating monitoring devices in the patient's house, it may be useful in our environment.

All in all, the aforementioned approach aims to solve the difficulties of device replacements and upgrades that usually lead to reconsidering the design of the whole system or, at least, to the modification of important software coding or hardware development. By applying these standards, communications between the different devices and the gateway will be transparent and universal no matter whom the vendor is.

\section{CONCLUSION}

The point-of-care described hereby has focused on usability concepts, aiming to develop intelligent and intuitive nodes, able to respond to the activities and situations recognized in the user's environment.

The primary objective of this platform is to examine whether a system of basic biomedical sensors (motion sensors, ecg, pulse oximetry, blood pressure, etc.) is able to detect behavioral patterns and help aging and ill populations with everyday activities and health care follow up. That is to say, to provide the foundation for an Ambient Intelligence approach.

Finally, a flexible, dynamic and easy to program architecture has been implemented, enabling software developers to focus on application level without paying attention to network and hardware levels.

\section{REFERENCES}

Ades PA, Pashkow FJ, Fletcher G, Pina IL, Zohman LR, Nestor JR. A controlled trial of cardiac rehabilitation in the home setting using electrocardiographic and voice transtelephonic monitoring. American Heart J ournal, vol 139, sup 3, pp 543-8. 2000.

Coleri S, Puri A, and Varaiya P. Power Efficient system for Sensor Networks. 8th IEEE International Symposium on Computers and Communication Proceedings (ISCC 2003). 2003

Cucchiara R, Grana C, Prati A, Vezzani R. Probabilistic posture classification for Humanbehavior analysis. Systems, Man and Cybernetics, Part A, IEEE Transactions on, vol. 35, sup. 1, pp 42-54, 2005 (ISSN: 1083-4427).

Heinzelman WB, Murphy AL, Carvalho HS and Perillo MA. Middleware to Support Sensor Network Applications. IEEE Network Magazine Special Issue. 2004

Hernandez C, Casas A, Escarrabill et al. Home hospitalization of exacerbated COPD patients. A randomized controlled trial of clinical efficacy and costs of services for chronically ill patients. ERJ vol 21, sup 1, pp 58-67. 2003

Information Society Technologies Advisory Group. ISTAG, Scenarios for Ambient Intelligence in 2010. http:// www. cordis. lu/ istag. htm 2002 
Jimenez-Fernandez S, Araujo-Pinto Á, Cobo-Sánchez de Rojas A, del Pozo-Guerrero F, NietoTaladriz O, de Toledo-Heras P \& Moya-Fernández J M. PERSEIA: a Biomedical Wireless Sensor Network to Support Healthcare Delivery for the Elderly and Chronically III. 28th IEEE EMBS Annual International Conference, pp. 2064-2066. 2006. (ISBN: 14244-0033-3, ISSN: 1557-170X)

Morlion B, Knoop C, Paiva M, Estenne M. Internet-based home monitoring of pulmonary function after lung transplantation. American Journal of Respiratory and Critical Care Medicine vol 165, sup 5, pp 694-697. 2002.

Trivedi MM, Huang KS, Mikic I. Dynamic context capture and distributed video arrays for intelligent spaces. Systems, Man and Cybernetics, Part A, IEEE Transactions on, vol. 35, sup. 1, pp 145-163, 2005 (ISSN: 1083-4427).

Ranganathan A, and Campbell RH. A middleware for context-aware agents in ubiquitous computing environments. Proceedings of the International Middleware Conference, volume 2672 of Lecture Notes in Computer Science, 143-161. Springer Berlin / Heidelberg. 2003

Rabaey JM et al. PicoRadio supports ad hoc ultra-low power wireless networking. IEEE Computer, 33, 7, pp 42-48. 2000

Remagnino P, Foresti GL. Ambient Intelligence: A New Multidisciplinary Paradigm. Systems, Man and Cybernetics, Part A, IEEE Transactions on, vol. 35, sup. 1, pp 1-6, 2005 (ISSN: 10834427)

Sgroi M, Wolisz A, Sangiovanni-Vincentelli A and Rabaey J M. A service-based universal application interface for ad-hoc wireless sensor networks whitepaper. U.C. Berkeley. 2004

\section{TERMS AND DEFINITIONS}

Home Area Network (HAN). The interconnection of information technology devices within the range of a house.

Embedded system. Special-purpose system in which the computer is completely encapsulated by or dedicated to the device or system it controls.

Home care. Health care given to a person in his/ her own home that aims to enable people to remain at home rather than use residential, long-term, or institutional-based nursing care.

LR-WPAN. Low Rate Wireless Personal Area Network.

Point-of-care. Care at the bedside or anywhere else where the patient might be, or where the caregiver makes the decisions.

Usability. Perception of a target user of the effectiveness (fit for purpose), efficiency (work or time required to use) and ease of use of a device, interface, computer program, web site, etc.

Wearable sensors. Biomedical sensors that, due to their small size and weight, can be worn on the body.

Wireless communication. Communication that takes place via airwaves as opposed to cables. 